\title{
LEVANTAMENTO FLORÍSTICO DA ÁREA DE PROTEÇÃO AMBIENTAL DE JERICOACOARA, CEARÁ
}

\author{
Lígia Queiroz Matias² \\ Edson Paula Nunes ${ }^{2}$
}

Recebido em 6/10/1999. Aceito em 17/8/2000

\begin{abstract}
RESUMO - (Levantamento florístico da Área de Proteção Ambiental de Jericoacoara, Ceará). Estudou-se a composição florística da Área de Proteção Ambiental de Jericoacoara, Estado do Ceará, nordeste do Brasil (247' $2^{\circ} 51^{\prime} \mathrm{S}$ e $40^{\circ} 36^{\prime}-40^{\circ} 24^{\prime} \mathrm{W}$ ). O clima da região é do tipo Aw' (segundo a classificacação de Köppen) com temperaturas médias oscilando entre $35^{\circ} \mathrm{C}$ e $22^{\circ} \mathrm{C}$, com chuvas de verão (dezembro a junho), possuindo um período de estiagem de 5 a 6 meses, frequentemente interrompido por chuvas ocasionais em setembro e outubro, e apresenta índice xerotérmico de Gaussen variável entre 100 e 150. As formações vegetacionais estudadas foram: vegetação com influência flúvio-marinha ou manguezal em estuários e na região de pós-praia e vegetação com influência marinha ("restingas"). Foram identificadas 87 espécies, 77 gêneros e 39 famílias. As famílias que apresentaram maior riqueza específica foram: Poaceae (13\%), Cyperaceae (13\%), Fabaceae (6\%), Amaranthaceae (6\%) e Rubiaceae (6\%). Alguns aspectos relacionados a conservação ambiental na APA são discutidos.
\end{abstract}

Palavras-chave - flora litorânea, psamófitos, vegetação de dunas, planície costeira, APA Jericoacoara

ABSTRACT - (Floristic inventory of the Jericoacoara Environmental Protected Area, Ceará). The flora of Jericoacoara Environmental Protected Area was studied in the State of Ceará, northeast of Brasil $\left(2^{\circ} 47^{\prime}-2^{\circ} 51^{\prime} \mathrm{S}\right.$ e $40^{\circ} 36^{\prime}-40^{\circ} 24^{\prime} \mathrm{W}$ ). The Aw' type climate (Köppen classification) has mean temperatures of $22^{\circ}-35^{\circ} \mathrm{C}$ with rains in summer (December to June). There is a hot, dry period of 5-6 months from July to December and sporadical rains occurs in September and October. Gaussen's xerothermic index oscilates between 100 to 150 . The main vegetation types are: the mangroves on the stuaries and beaches, the creeping psammophyte vegetation on sand dunes with aquatic macrophytes in the slacks, and the xeromorphic scrub. Eighty-seven species belonging to forthy-one families and seventy-seven genera were identified. Poaceae (13\%), Cyperaceae (13\%), Fabaceae (6\%), Amaranthaceae $(6 \%)$ e Rubiaceae $(6 \%)$ were the most species-rich families. Aspects of environmental conservation within the Jericoacoara Environmental Protection Area are discussed.

Key words - coastal plants, psammophytes, sand dune vegetation, sand coastal plain, Jericoacoara Protection Area

1 Desenvolvido com auxílio da Fundação Cearense de Amparo à Pesquisa (FUNCAP)

2 Departamento de Biologia, Centro de Ciências, Universidade Federal do Ceará, CEP 60970-451, Fortaleza, CE, Brasil 


\section{Introdução}

O litoral nordestino compreendido entre o Delta do Rio Parnaíba (Piauí) e o Cabo de São Roque (Rio Grande do Norte) possui clima semiárido com predomínio de grandes extensões de campos de dunas (Araújo 1992; Lacerda et al.1993).

Nesta faixa, a área litorânea cearense oferece continuidade à suave declinação do pedimento rochoso que se inicia na base das chapadas periféricas e dos sopés dos relevos residuais da depressão sertaneja. Seu traço comum mais característico é a presença contínua, ao longo da costa, dos tabuleiros de sedimentos arenosos de origem Quaternária ou dunas. A vegetação litorânea apresenta uma diversidade fisionômica, expressando uma composição que geralmente mescla espécies próprias do litoral com outras provenientes das matas vizinhas, das caatingas, além de diversas do cerrado (Fernandes \& Gomes 1975). Os agrupamentos vegetacionais apresentam-se como vegetação das planícies litorâneas, das dunas, dos tabuleiros litorâneos e das planícies flúvio-marinhas, além das macrófitas aquáticas das lagoas (Fernandes 1990). Na faixa litorânea do Ceará, as formações vegetais de maior significado fitoecológico e de representatividade, como conjunto, são a vegetação pioneira psamófila, a vegetação subperenifolia de dunas e a vegetação perenifolia paludosa marítima de mangue (Vicente da Silva 1998).

A APA de Jericoacoara situa-se na região definida como "Complexo Edáfico de Primeira Ocupação ou formações pioneiras" (Veloso et al. 1991), caracterizada por terrenos instáveis cobertos por uma vegetação em constante sucessão.

Trata-se de uma faixa de terra que acompanha paralelamente a faixa costeira, com largura de 5 a $10 \mathrm{~km}$ e constituída por sedimentos arenosos recentes, intensamente trabalhados pela ação eólica. Na faixa costeira observa-se, às vezes, a presença de inúmeras formações litológicas de maior resistência, associados que estão, aos afloramentos de quartizito ou às concreções lateríticas. Tanto estas como os paredões costeiros esculturados pela abrasão marinha, responsável pelas falésias, ocorrem na APA de Jericoacoara (Souza et al. 1979; Souza 1988).

Considerando a geomorfologia presente na APA, Fernandes (In Georgen 1985) descreveu as seguintes unidades particulares da paisagem: serrote, restinga graminea, complexo de dunas migratórias com lagoas rasas permanentes, lagoas rasas periódicas e lagoas profundas permanentes, o tabuleiro, o manguezal, os gramados halofíticos e as vastas praias arenosas. Em seu estudo florístico indica 73 espécies como elementos principais dos diversos tipos vegetacionais.

O presente trabalho teve como objetivo o levantamento da flora da APA de Jericoacoara, caracterizando-a segundo os tipos vegetacionais citados acima por Veloso et al.

\section{Material e métodos}

A Área de Proteção Ambiental de Jericoacoara ou APA de Jericoacoara, criada através do Decreto Federal n. 90.379, de 29/10/ 84 (Fig. 1), localiza-se no litoral do Estado do Ceará $\left(2^{\circ} 47^{\prime}-2^{\circ} 51^{\prime} \mathrm{S}\right.$ e $\left.40^{\circ} 36^{\prime}-40^{\circ} 24^{\prime} \mathrm{W}\right)$ e possui 5.480ha.

O clima da região é do tipo quente e úmido, com chuvas de verão (Aw'sg. Köppen) e com temperaturas médias oscilando entre $35^{\circ} \mathrm{C}$ e $22^{\circ} \mathrm{C}$, apresentando estação seca com média de duração de 5 a 6 meses, frequentemente interrompida por chuvas ocasionais em setembro e outubro. O índice xerotérmico de Gaussen varia entre 100 e 150 (Georgen 1985). Como ambiente litorâneo, diferencia-se do interior do estado por possuir clima mais úmido e uma intensa ação eólica, com ventos predominantes no sentido E-NE que influencia a dinâmica da geomorfogênere local (Vicente da Silva 1998).

Os sedimentos areno-quartzosos predominam e formam a faixa praial, pós-praia e 


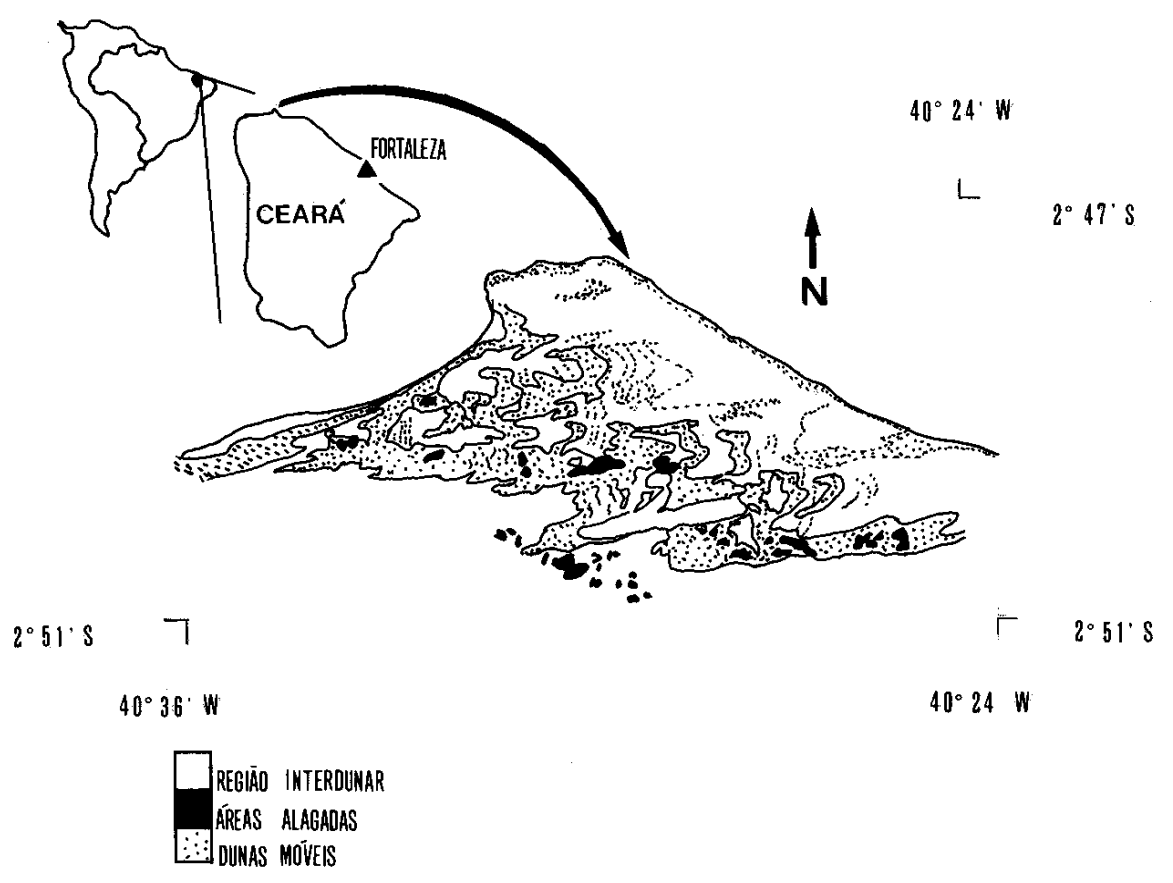

Figura 1. Localização da Área de Proteção Ambiental de Jericoacoara, Ceará. (Adaptado de Georgen 1985, escala aproximada 1:70.000)

campo de dunas e, recortando-os, há pequenos cursos d'água onde aparece um substrato silto-argiloso. Estes sedimentos mais recentes recobrem a Formação Barreiras, que por sua vez, está sobreposta a rochas cristalinas do Pré-Cambriano. Parte destas rochas são compostas por quartizito ferrífero e afloram ao norte da APA, formando o Serrote de Jericoacoara com altitude de $90 \mathrm{~m}$. Em áreas de relevo ondulado a forte ondulado (Serrote), ocorre predomínio de solos formados por areia quartzosa marinha eutrófica, os quais apresentam a baixa fertilidade natural e a drenagem excessiva como principais limitações ao desenvolvimento da vegetação. Em áreas de mangue, ocorrem solos halomórficos, pouco consolidados e semifluidos (Georgen 1985; IPLANCE 1995) .

Para determinação da composição florística foram realizadas coletas aleatórias das espécies vegetais de cada unidade de vegetação, sendo elas: vegetação com influência flúvio-marinha ou manguezal e vegetação com influência mari- nha ("restingas"). Convém ressaltar que a formação geomorfológica presente na APA de Jericoacora não constitui uma restinga sensu Guerra (1978), mas sim um campo de dunas, enquadrando-se também, no sentido empregado por Rizzini (1997).

A coletas ocorreram mensalmente no primeiro semestre de 1997 e de 1998, coincidindo com o período de maior precipitação na região. O material foi herborizado conforme as técnicas usuais em botânica (Mori et al. 1985; Bridson \& Forman 1998) e depositado no Herbário EAC (Herbário Prisco Bezerra, Departamento de Biologia, Centro de Ciências, Universidade Federal do Ceará). O metodo de coleta para macrófitos aquáticos segue as orientações de Haynes (1984) e Pedralli (1990). A identificação do material foi realizada através da utilização da literatura botânica disponível, comparação com material de herbário e envio de exsicatas para especialistas para confirmação das identificações.

A listagem do material encontrado foi ela- 
borada segundo o sistema de classificação de Cronquist (1988), sendo apresentada em ordem alfabética por famílias, gêneros e espécies. Inclui-se dados sobre o hábito das plantas, seguindo a nomenclatura de Font Quer (1975), para as espécies terrestres: Arb arbóreo; Abt - arbustivo, Her - herbáceo, Li liana e Cook (1974) para os macrófitos aquáticos - MA.

\section{Resultados e discussão}

Foram identificadas 87 espécies, 77 gêneros e 41 famílias (Tab. 1), número considerado expressivo tendo em vista os levantamentos realizados por Fernandes (1985) para a APA de Jericoacoara e para formações de praias e dunas de diferentes localidades como Tavares/RS (Danilevicz 1989), Natal/RN (Freire 1990), Florianópolis/SC (Souza et al. 1991/1992), São Luís/MA (Freire \& Monteiro 1993), Mataraca/ PB (Oliveira-Filho e Carvalho 1993), Cabo Frio/ RJ (Cordeiro 1998) e Marambaia/RJ (Menezes \& Araújo 1999).

As famílias que apresentaram maior riqueza específica foram: Poaceae (13\%), Cyperaceae (13\%), Fabaceae (6\%), Amaranthaceae $(6 \%)$ e Rubiaceae (6\%). Se consideradas em conjunto, as famílias mais representadas perfazem $43 \%$ da flora da APA de Jericoacoara.

Observou-se o predomínio de formações pioneiras herbáceas, constituída por espécies predominantemente psamófila-reptantes.

Principais fisionomias e espécies ocorrentes

Vegetação com influência flúvio-marinha ou manguezal - Como principais espécies vegetais arbóreas consideradas obrigatórias nos manguezais cearenses, estão Rhizophora mangle L. (mangue-vermelho), Laguncularia racemosa (L.) Gaertn. (mangue-manso, branco ou rajadinho), Avicennia germinans (L.) Stearn (canoé, mangue-preto ou síriba) e Conocarpus erecta $\mathrm{L}$. (mangue-ratinho ou botão), as quais ocorrem na foz de riachos que cortam a APA, constituindo mangues do tipo ribeirinho.
Em áreas interiores dos manguezais, desprovidas de árvores, há o predomínio de espécies herbáceas, destacando-se Batis maritima L. (bredo-do-mangue) e Sesuvium portulacastrum L. (beldroega).

Aquíferos subterrâneos dos campos de dunas ocorrem em algumas localidades da região de pós-praia, propiciando a formação de manguezal tipo franja, com aspecto fisionômico semelhante ao descrito por Cintrón \& SchafferNovelli (1983); Lugo \& Snedaker (1974); Tomlinson (1994) sendo constituído, predominantemente, por $R$. mangle $\mathrm{L}$.

Vegetação com influência marinha ("restingas") - Esta formação possui como principais espécies: Ipomoea asarifolia Roem. \& Schult (salsa), Remirea maritima Aublet (pinheirinho-da-praia), Richardia grandiflora (Cham. \& Schlecht.) Steud. (barba-de-bode), Heliotropium lanceolatum Ruiz. \& Pav. (cristade-galo), Blutaparon portulacoides (A. St. Hil.) Miers (bredo-da-praia), Sesuvium portulacastrum L. (beldroega) e diversas leguminosas e gramíneas. Essa vegetação adquire funções estabilizadoras iniciais nas áreas de pós-praia e campo de dunas, na fixação do substrato arenoso, contribuindo nos processos de pedogênese, através do aporte de matéria orgânica e da retenção de umidade no substrato (Vicente da Silva 1998).

Em áreas interdunares alagadas, destacamse algumas ciperáceas e outras macrófitas aquáticas como Nymphoides indica (L.) O. Kuntze (aguapé-da-flor-miúda) e Typha domingensis Pers. (taboa).

Quando as condições edáficas tornam-se mais favoráveis, pequenos arbustos como o murici (Byrsonima spp.) vão ocupando áreas da pós-praia e regiões interdunares, dando início ao desenvolvimento de uma vegetação de porte arbustivo. Esta vegetação apresenta-se sob forma de moitas abertas ou moitas fechadas, com a presença Chrysobalanus icaco L. (guajiru), Abrus precatorius L. (jeriquiti) e Anacardium occidentale L. (cajueiro). 
Tabela 1. Relação das espécies de angiospermas encontradas na Área de Proteção Ambiental de Jericoacoara, CE. Os números de coleção referem-se ao registro no Herbário Prisco Bezerra (EAC). Hábitos: Arb = arbóreo, Abt = arbustivo, Her = herbáceo, $\mathrm{Ma}=$ macrófito aquático, $\mathrm{Li}=$ Liana .

\begin{tabular}{lll}
\hline Famílias e espécies & Coleção & Nomes vernaculares Hábito \\
\hline
\end{tabular}

Aizoaceae

Sesuvium portulacastrum $\mathrm{L}$.

2682926139

Alismataceae

Echinodorus tenellus (Martius) Buchenau

Amaranthaceae

Alternantera litoralis var. maritima (Mart.) Pedersen 26919

Alternanthera brasiliana Kuntze

Froelichia lanata Moq.

Gomphrena sp.

Blutaparon portulacoides (A. St.-Hil.) Miers

Anacardiaceae

Anacardium occidentale $\mathrm{L}$.

Apocynaceae

Allamanda blanchetii A. DC.

Aspidosperma pyrifolium Mart.

Asteraceae

Porophyllum latifolium Benth.

Stilpnopappus trichospiroides Mart.

Bidens sp.

Avicenniaceae

Avicennia germinans (L.) Stearn

Batidaceae

Batis maritima $\mathrm{L}$.

Heliotropium lance
Tournefortia sp.

Caesalpinaceae

Bauhinia pentandra (Bong.) Vogel ex Steud. 25006

Caesalpinia bracteosa Tul.

Chamaecrista ramosa var. ramosa Irwin \& Barneby

Cactaceae

Cereus jamacaru A. DC.

Capparaceae

Capparis cynophallophora $\mathrm{L}$.

Chrysobalanaceae

Chrysobalanus icaco L.

Commelinaceae

Commelina nudiflora $\mathrm{L}$.

Combretaceae

Conocarpus erectus $\mathrm{L}$.

Laguncularia racemosa (L.) Gaertn.

Convolvulaceae

Evolvulus ovatus Fernald

Ipomoea asarifolia Roem. \& Schultz

Jacquemontia hirtusa Chois

Cucurbitaceae

Cucumis anguria $\mathrm{L}$.
2683026832

26918

26926

25016

26921

28.507

26840

25002

2684126845

2502526842

26849

28160

24950

24987

25019

25010

25009

2500726852

24998

24986

27622

24952

2495126140

26843

25005

27624

Beldroega-da-praia Her

Ma

Periquito-da-praia Her

Quebra-panela Her

Cabeça-branca Her

Cabeça-branca Her

Bredinho Her

Cajueiro Arb

Alamanda-roxa $\quad$ Abt

Pereiro Abt

Cravo-de-urubu Her

Her

Margaridinha $\quad$ Her

Mangue negro Arb

Bredoeiro Her

Crista-de-galo Her

Her

Capa-bode

Catingueira

Abt

Abt

Her

Mandacaru $\quad$ Abt

Feijão Branco $\quad$ Abt

Guajiru

Arb

Marianinha Her

Mangue-ratinho Arb

Mangue-branco Arb

Salsa

Her

Jitirana Her

26347

Maxixe-do-mato Her 
Tabela 1. (continuação)

Famílias e espécies

Coleção

Cyperaceae

Bulbostylis capilaris (L.) Kunth ex Clarke

Cyperus amabilis Vahl

Cyperus liguralis $\mathrm{L}$.

Cyperus maritimus Poir.

Eleocharis atropurpurea (Retz.) J. \& C. Presl

Eleocharis geniculata (L.) Roem. \& Schult.

Fimbristylis spadicea (L.) Vahl

Fimbristylis cymosa $\mathrm{R}$. Br.

Kyllinga vaginata Lam.

Remirea maritima Aublet

Rhynchospora riparia (Nees) Boeck.

Euphorbiaceae

Cnidosculus urens (L.) Arthur

Chamaesyce hyssopifolia (L) Small

Jatropha mollissima (Pohl.) Baill.

Fabaceae

Abrus precatorius $\mathrm{L}$.

Indigofera microcarpa Desv.

Macroptilium panduratum Marechal \& Baudet

Stylosanthes viscosa $\mathrm{Sw}$.

Zornia orbiculata Mohl..

Lamiaceae Marsypianthes chamaedrys (Vahl.) Kuntze

Malpighiaceae

Byrsonima crassifolia Hum. Bonp. \& Kunth

Byrsonima verbascifolia Rich.

Malvaceae

Pavonia cancellata Cav.

Menyanthaceae Nymphoides indica (L.) O. Kuntze

Mimosaceae

Mimosa misera Benth.

Molluginaceae

Mollugo verticillata $L$.

Myrtaceae

Psidium sp.

Najadaceae

Najas marina $\mathrm{L}$.

Olacaceae

Ximenia americana $\mathrm{L}$.

Passifloraceae

Passiflora foetida $\mathrm{L}$.

Passiflora subrotunda Mart.

Poaceae

Andropogon selloanus (Hack.) Hack.

Antephora hermafrodita (L.) Kuntze

Cenchrus echinatus L.

Digitaria ciliaris (Retz.) Koeler

Dactyloctenium aegyptium (L.) Willd.

Eragrostis ciliaris (L.) R. Br.

Panicum trichoides Swartz

2750327504
27505
27508
2501327016
2701127512
27510
25020
2501425015
27509
28152
27506

24995
26345
24996
24988
2684824989
24990
26928
25276

27014

2499925001

25023

26850

25995

26844

26348

25008

26349

Capim

Cipó-da-praia

Alecrim-da-praia Her

Capim junco

Capim-açu

Her

Her

Her

Ma

$\mathrm{Ma}$

Her

Her

Her

Her

Cansação

Erva-de-leite

Pinhão

Abt

Her

Abt

Jiriquiti

$\mathrm{Li}$

Anil

Her

Her

Her

Urinána

Her

Murici

Murici

Abt

Abt

Malva-rateira

Her

Ma

Her

Molungo

Her

Arb

Ma

25018

Ameixa

Abt

25003

25000

Maracujá-de-estalo

Maracujá

$\mathrm{Li}$

$\mathrm{Li}$

12875

2681826846

2684726820

26900

2681226816

26855

Capim

Her

Capim-mimoso Her

Carrapicho Her

Capim-milhá Her

Pé-de-Galinha Her

Capim

Her

26815268172681126813 Capim

Her 
Tabela 1. (continuação)

\begin{tabular}{|c|c|c|c|}
\hline Famílias e espécies & Coleção & Nomes vernaculares & Hábito \\
\hline Paspalum plicatulum Michx. & 27623 & Capim & Her \\
\hline Paspalum scutatum Nees ex. Trin. & 2681426821 & Capim & Her \\
\hline Paspalum vaginatum Swartz & 24849 & Capim & Her \\
\hline Sporobolus virginicus (L.) Kunth & 24953268192682226839 & Capim & Her \\
\hline \multicolumn{4}{|l|}{ Polygalaceae } \\
\hline \multirow{2}{*}{\multicolumn{4}{|c|}{ Pontederiaceae }} \\
\hline & & & \\
\hline \multicolumn{3}{|l|}{ Potamogetonaceae } & Ma \\
\hline Potamogeton ferrugineus Hagström & 26924 & & $\mathrm{Ma}$ \\
\hline \multicolumn{4}{|l|}{ Rhizophoraceae } \\
\hline Rhizophora mangle $\mathrm{L}$. & 27583 & Mangue-vermelho & Arb \\
\hline \multicolumn{4}{|l|}{ Rubiaceae } \\
\hline Borreria verticillata $($ L.) G. F. W. Meyer & 250042634626836 & Vassourinha-de-botão & Her \\
\hline Diodia rigida (R. \& S.) Cham. \& Schlecht. & 26831 & Vassourinha & Her \\
\hline Diodia teres Walter & 26833268342683526838 & Vassourinha & Her \\
\hline $\begin{array}{l}\text { Mitracarpus frigidus (R. \& S.) } \\
\quad \text { var. discolor (Miq.) K. Schum. }\end{array}$ & 26837 & & Her \\
\hline $\begin{array}{l}\text { Richardia grandiflora (Cham. \& Schlecht.) Steud. } \\
\text { Scrophulariaceae }\end{array}$ & 25017 & Capim-barba-de-bode & Her \\
\hline Bacopa monnieri (L.) Pennell & 27004 & Alecrim-do-brejo & Her \\
\hline \multicolumn{4}{|l|}{ Solanaceae } \\
\hline Melananthus sp. & 25021 & & Abt \\
\hline Solanum paludosum Moric. & 2499325012 & & Abt \\
\hline \multicolumn{4}{|l|}{ Turneraceae } \\
\hline Turnera subulata $\mathrm{Sm}$. & $\begin{array}{l}24997 \\
27013\end{array}$ & Chanana & Her \\
\hline \multicolumn{4}{|l|}{ Typhaceae } \\
\hline Typha domingensis Pers. & 20665 & Taboba & Ma \\
\hline Hybanthus ipecacuanha Bail. & 2685126853 & Ipecacuanha & Her \\
\hline
\end{tabular}

Uma vegetação arbustivo-arbórea mais densa ocupa as áreas protegidas da ação direta dos ventos, constituindo uma formação vegetal de maior porte, a qual limita-se a uma formação insular na face norte do Serrote. Fernandes (1985) descreve "essa vegetação lenhosa de uma composição mista de elementos do cerrado e da caatinga, podendo ser considerada, em resumo, como um sertão. A característica significativa é a poda natural do vento, que limita o crescimento vertical da vegetação ...”.

Na composição dessa vegetação arbustivoarbórea que varia de 2 a 4 metros de altura, as principais espécies vegetais são: Byrsonima crassifolia
Humb. Bonp. \& Kunth. (murici), Chrysobalanus icaco L. (guajiru), Anacardium occidentale L. (cajueiro) e Cereus jamacaru A. DC. (mandacaru).

A fisionomia da vegetação da APA de Jericoacoara assemelha-se à descrita Cordazzo \& Costa (1989) para dunas em Garopaba/SC; por Freire (1990) para formações das praias e do sopé das dunas em Natal/RN e por Menezes \& Araújo (1999) para comunidades de ante-duna da restinga de Marambaia/RJ. Porém, a composição florística destas comunidades são muito distintas, com exceção para as espécies Remirea maritima Aublet e Sporolobus virginicus (L.) 
Kunth que são comumente encontradas na costa brasileira. Este fato pode estar de acordo com Menezes \& Araújo op. cit. quando afirma que a flora costeira apresenta certo grau de semelhança especialmente quanto as espécies dominantes, porém revela diversidade significativa de espécies menos abundantes.

Tomando como parâmetro as famílias mais representadas na APA de Jericoacoara, ou seja, Cyperaceae, Poaceae, Rubiaceae, Fabaceae e Amaranthaceae, $40 \%$ das espécies são anuais, tomando como base as descrições de Bacigalupo (1974,1993), Renvoize (1984), Luceño \& Alves (1997), Luceño et al. (1997), Lorenzi (1982) e Souza (1997). A presença significativa de espécies anuais podem estar associadas não apenas ao intenso dinamismo ambiental mas também pode ser um reflexo do uso do solo na APA, onde predomina o pastoreio (Matias e Vicente da Silva 1998). Filgueiras (1991), analisando a família Poaceae em áreas alteradas, observou uma pressão seletiva para espécies com forma de vida críptica. Estudos específicos poderão melhor esclarecer esta questão, pois, além da tendência à anualidade como forma de vida, observou-se que $20 \%$ das espécies herbáceas coletadas na APA de Jericoacora são citadas na literatura como indicadoras de ambientes alterados por ação antrópica (Luceño \& Alves 1997; Luceño et al. 1997; Renvoize 1984).

\section{Agradecimentos}

Ao IBAMA pelo apoio local; a todos os moradores que de uma forma ou de outra contribuíram para a realização deste trabalho; à Bárbara Machado, Cândido Cunha, Hilder Magalhães e Hélbia Nakakura, alunos da UFC, pelo apoio aos trabalhos de campo; ao prof. Elnatan Bezerra (UVA-CE) pela identificação das rubiáceas e Prof. Marccus Vinicius Alves (UFPE) pela determinação das ciperáceas.

\section{Referências bibliográficas}

Araújo, D .S. D. 1992. Vegetation types of sandy coastal plains of tropical Brazil: a first aproximation. Pp. 337-347. In: U. Seeliger (Ed.), Coastal Plant Communities of Latin America. Academic Press, London.

Bridson, D. \& Forman, L. 1998. The Herbarium Handbook. Royal Botanical Garden, Kew.

Bacigalupo, N. M. 1974. Rubiaceae. Pp. 3-50. In: A. Burkart, Flora Ilustrada de Entre Rios, vol.6. INTA, Buenos Aires.

Bacigalupo, N. M. 1993. Rubiaceae. Pp. 375-437. In: A. L. Cabrera, Flora de la Provincia de Jujuy, vol.13 INTA, Buenos Aires.

Cintrón, G. \& Schaeffer-Novelli, Y. 1983. Introduccion a la Ecologia del Manglar. UNESCO, Uruguai.

Cook, C. D. K. 1974. Water Plants of the World. W. Junk, Hague.

Cordazzo, C. V. \& Costa, C. S. B. 1989. Associações vegetais das dunas frontais de Garopaba (SC). Ciência e Cultura 41(9): 906-910.

Cordeiro, S. Z. 1998. Análise da Cobertura Vegetal em Três Áreas de Topografia Distinta na Praia do Peró, Cabo Frio, RJ. Disseração de Mestrado. Universidade Federal do Rio de Janeiro, Rio de Janeiro.

Cronquist, A. 1988. The Evolution and Classification of Flowering Plants. The New York Botanical Garden, New York.

Danilevicz, E. 1989. Flora e vegetação de restinga na Barra da Laguna do Peixe, Tavares, Rio Grande do Sul: levantamento preliminar. Iheringia Série Botânica 39: 69-79.

Fernandes, A. \& Gomes, M. A. F. 1975. Plantas de cerrado no litoral cearense. In: Annais do XXVI Congresso Nacional de Botância. Rio de Janeiro. 1975. Academia Brasileira de Ciências, Rio de Janeiro.

Fernandes, A. 1985. Aspectos biológicos e ecológicos. Pp. 85-89. In: J. Georgen (Coord.), Área de Proteção Ambiental Jericoacoara. PRINTER/CE, UECE/NUGA, SUDEC/DRN, PRMA/DF, PMA/ CE, CEDCT/CE, GTZ, Fortaleza.

Fernandes, A. 1990. Temas Fitogeográficos. Stylus Comunicações, Fortaleza.

Filgueiras, T. S. 1991. Desertificação em Gilbués, Piauí; uma análise agrostológica. Cadernos de Geociências 7: 23-27.

Font Quer, P. 1975. Diccionario de Botánica. Ed. Labor, Barcelona.

Freire, M. C. C. \& Monteiro, R. 1993. Florística das praias da ilha de São Luís, Estado do Maranhão (Brasil): 
diversidade de espécies e suas ocorrências no litoral brasileiro. Acta Amazônica 23(2-3): 125-140.

Freire, M. S. B. 1990. Levantamento florístico do Parque Estadual das Dunas de Natal. Acta Botânica Brasilica 4(2/Supl.): 41-59.

Georgen, J. 1985. Área de Proteção Ambiental Jericoacoara. PRINTER/CE, UECE/NUGA, SUDEC/DRN, PRMA/DF, PMA/CE, CEDCT/CE, GTZ, Fortaleza.

Guerra, A. T. 1978. Dicionário Geológicogeomorfológico. IBGE, Rio de Janeiro.

Haynes, R. R. 1984. Techniques for collecting aquatic and marsh plants. Annals of Missouri Botanical Garden 71: 229-231.

IPLANCE. 1995. Atlas do Ceará. Edições IPLANCE, Fortaleza.

Lacerda, L. D.; Araújo, D. S. \& Maciel, N. C. 1993. Dry coastal ecosystems of the tropical brazilian coast. Pp. 477-493. In: E. van der Maarel (Ed.), Dry Coastal Ecosystems. Africa, America and Oceania. Elsevier, Amsterdam.

Lorenzi, H. 1982. Plantas Daninhas do Brasil. Ed. do Autor, Nova Odessa.

Luceño, M. \& Alves, M. V. 1997. Clave de los géneros de ciperáceas de Brasil y novedades taxonómicas e corológicas en la familia. Conservatoire et Jardin Botaniques de Genéve 52(1): 185-197.

Luceño, M.; Alves, M. V. \& Mendes, A. P. 1997. Catálogo florístico y claves de identificaión de las ciperáceas de los estados de Paraíba y Pernambuco (Nordeste de Brasil). Anales Jardín Botánico de Madrid 55(1): 67-100.

Lugo, A. E. \& Snedaker, S. C. 1974. The ecology of mangroves. Annual Review of Ecology Systematics 5: 39-64.

Matias, L. Q. \& Vicente da Silva, E. 1998. Estudo da vegetação da Área de Proteção Ambiental de Jericoacoara, Ceará. Relatório técnico-IBAMA. UFC, Fortaleza.
Menezes, L. F. T. \& Araújo, D. S. D. 1999. Estrutura de duas formações vegetais do cordão externo da restinga de Maranbaia, RJ. Acta Botanica Brasílica 13(2): 115-236.

Mori, S. A.; Mattos-Silva, L . A.; Lisboa, G. \& Coradin, L. 1985. Manual de Manejo do Herbário Fanerogâmico. 2a ed. CEPLAC, Ilhéus.

Oliveira-Filho, A. T. \& Carvalho, D. A. 1993. Florística e fisionommia da vegetação no extremo norte do litoral da Paraíba. Revista Brasileira de Botânica 16(1): 115-130.

Pedralli, G. 1990. Macrófitos aquáticos: técnicas e métodos de estudos. Estudos de Biologia 26: 524.

Renvoize, S. A.1984. The grasses of Bahia. Royal Botanical Garden, Kew.

Rizzini, C. T. 1997. Tratado de fitogeografia do Brasil: Aspectos e Ecológicos, Sociológicos e Florísticos. Âmbito Cultural Ed., Rio de Janeiro.

Souza, E. B. 1997. Estudos taxonômicos dos gêneros Staelia Cham. \& Schltdl. e Mitracarpus Zucc. ex. Roem. \& Schult. (Spermacoceae-Rubiaceae) no Estado de Pernambuco - Brasil. Dissertação de Mestrado. Universidade Federal Rural de Pernambuco, Recife.

Souza, M. J .N. 1988. Contribuição ao estudo das unidades morfo-estruturais do estado do Ceará. Revista de Geologia 1: 73-91.

Souza, M. J. N.; Lima, F. A. M. \& Paiva, J. B. 1979. Compartimentação topográfica do estado do Ceará. Ciência Agronômica 9(1-2): 77-86.

Souza, M. L. D. R.; Falkenberg, D. B.; Amaral, L. G.; Fronza, M.; Araujo, A. M. \& SÁ, M. R. 1991/1992. Vegetação do pontal da Daniela, Florianópolis, SC, Brasil. I. Levantamento florístico e mapa fitogeográfico. Insula 21: 87-117.

Tomlinson, P. B. 1994. The Botany of Mangroves. Cambridge University Press, Cambridge.

Veloso, H. P.; Rangel Filho, A. L. \& Lima, J. C. A. 1991. Classificação da vegetação brasileira, adaptada a um sistema universal. IBGE, Rio de Janeiro.

Vicente da Silva, E. 1998. Geoecologia da Paisagem do Litoral Cearense; uma abordagem ao nível de escala regional e tipológica. Tese para concurso de Professor Titular do Departamento de Geografia. Universidade Federal do Ceará, Fortaleza. 